\title{
Estrategias de afrontamiento en cuidadoras de personas con alzhéimer. Influencia de variables personales y situacionales
}

\author{
Coping strategies in caregivers of people with Alzheimer's. Influence of \\ personal and situational variables
}

\begin{abstract}
Resumen
El cuidado de una persona con alzhéimer ha sido identificado como una tarea altamente estresante para las cuidadoras informales. Las estrategias de afrontamiento permiten modular el impacto emocional que esta tarea provoca. El objetivo de este trabajo es describir las estrategias de afrontamiento más utilizadas por las cuidadoras informales de personas con alzhéimer e identificar si se presentan diferencias en la utilización de estas en función de variables personales y/o situacionales. Han participado 57 cuidadoras informales de personas con alzhéimer de una asociación rural de Castilla y León (España). La distinta utilización de las estrategias está relacionada de forma diferente con las variables estudiadas. No se aprecia un patrón de respuesta uniforme. El estudio confirma la idea de la flexibilidad del afrontamiento. Los resultados revelan la necesidad de formar en el manejo flexible y adaptativo de estas estrategias en función de los rasgos personales y las demandas específicas de la situación.
\end{abstract}

\section{Palabras clave}

Enfermedad de Alzheimer, estrategias de afrontamiento, estrés, carga del cuidador, demencia, alzhéimer.

\begin{abstract}
Caring for a person with Alzheimer's has been identified as a highly stressful task for the informal caregiver. The coping strategies allow modulating the emotional impact of this task. The purpose of this paper is to describe the coping strategies most commonly used by informal caregivers of people with Alzheimer's and to identify if there are differences in the use of these according to personal and-or situational variables. 57 informal caregivers of people with Alzheimer's from a rural association in Castilla y León (Spain) have participated. The different use of strategies is related in different ways to the variables studied. There is no uniform response pattern. The study confirms the idea of flexible coping. The results reveal the need for training in the flexible and adaptive management of these strategies according to personal traits and the specific demands of the situation.
\end{abstract}

\section{Keywords}

Alzheimer's disease, coping strategies, distress, caregiver burden, dementia, Alzheimer's.

\author{
Agustín Rodríguez Esteban \\ <arode@unileon.es> \\ Universidad de León. España \\ Leticia Pérez Álvarez \\ <leticiaperea@outlook.es> \\ Universidad de León. España
}

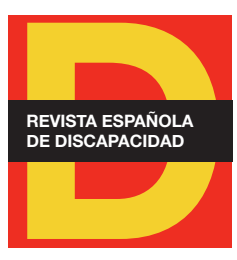

Para citar:

Rodríguez, A. y Pérez, L. (2019): "Estrategias de afrontamiento en cuidadoras de personas con alzhéimer. Influencia de variables personales y situacionales". Revista Española de Discapacidad, 7 (I): 153-171.

Doi: <https://doi.org/10.5569/23405104.07.01.08>

Fecha de recepción: 03-04-2018 Fecha de aceptación: 06-05-2019 


\section{Introducción}

Según datos de la Organización Mundial de la Salud (2016), cada año aparecen en el mundo 8 millones de casos nuevos de demencia, enfermedad que, en el año 2015, afectó a más de 47 millones de personas.

El alzhéimer es considerado como el tipo de demencia más común, representando en la actualidad entre un $60 \%$ y un $80 \%$ de estos casos (Alzheimer's Association, 2015). La mayor parte de los cuidados que reciben los personas con alzhéimer provienen del llamado cuidado informal, especialmente el que se desarrolla en el ámbito familiar (Bielsa, 2013; Mahdavi et al.; 2017; Rodríguez-González et al., 2017; Wennberg et al., 2015), y es prestado, en mayor medida, por mujeres (Coira y Bailon, 2014; Lorenzo et al., 2014; Manso et al., 2013)

Esta enfermedad presenta dos rasgos característicos que hacen que el cuidado sea una tarea altamente costosa para las cuidadoras ${ }^{1}$, especialmente desde el punto de vista emocional. En primer lugar, su carácter de cronicidad. En segundo lugar, el deterioro progresivo de las funciones cognitivas del enfermo, que hace que se vean afectadas sus capacidades para la realización de las actividades básicas de la vida diaria. La intensidad de la tarea del cuidado es, por tanto, elevada. Esta intensidad es difícilmente cuantificable, ya que el cuidado no se vincula tanto a actividades ligadas al rendimiento y a la productividad, sino que conforma una variable muy heterogénea ligada a los múltiples ritmos de la naturaleza y de las relaciones humanas (Carrasco, 2016; Durán, 2018; Ruiz-Adame et al., 2019).

Se utiliza así el constructo sobrecarga para referirse a aquella "carga emocional, física, social y económica que se produce como resultado de cuidar a un familiar o conocido con enfermedad crónica o discapacidad" (Rodríguez-González et al., 2017: 158). Entre los síntomas o problemas asociados a la sobrecarga se han documentado la depresión, la ansiedad, la soledad o, incluso, deficiencias en el sistema inmune (Bruvik et al., 2012; García-Alberca et al., 2012).

Pero el estrés ha sido, sin duda, la respuesta del organismo más estudiada. Se han desarrollado investigaciones de índole cuantitativa y cualitativa que describen los factores que pueden incidir en el mismo. Los resultados no han sido, sin embargo, plenamente coincidentes. Así, variables demográficas como el sexo, la relación de parentesco o el nivel educativo han aparecido como significativas en estudios cuantitativos como los de Adelman et al. (2014) o Manso et al. (2013). Investigaciones de índole cualitativa han encontrado también diferencias en el impacto de la carga y la capacidad de afrontamiento entre hombres y mujeres, así como cambios en la respuesta adaptativa según la etapa del cuidado (Daley et al., 2017; Martínez-Marcos y De la Cuesta-Benjumea, 2013; Moreno-Cámara et al., 2016; Rubio et al., 2017)

Sin embargo, estudios como los de McConaghy y Caltabiano (2005) o Raggi et al. (2015) no han reflejado relaciones de influencia en el caso de variables como el género o el nivel educativo. La influencia de variables clínicas, como el deterioro funcional o el número de horas dedicadas al cuidado, tampoco se ha mostrado consistente en la literatura (véanse al respecto los resultados de los citados estudios de Manso et al., 2013 o Raggi et al., 2015).

1. Dado el mayoritario número de cuidadoras mujeres, se utilizará el género femenino para referirse en general a las personas responsables del cuidado. 
No es posible, por tanto, establecer una relación directa entre todas estas variables y la respuesta emocional que se produce en las cuidadoras. Los resultados evidencian que lo importante es la variabilidad en la capacidad de estas para hacer frente a la tarea del cuidado de una persona con enfermedad de Alzheimer (Artaso et al., 2002).

Pearlin (1990), dentro del marco de referencia del modelo transaccional de estrés, presenta un proceso multidimensional según el cual el impacto negativo del cuidado en la salud mental de las cuidadoras depende de un conjunto de factores interrelacionados. Entre estos, enumera los factores o variables contextuales y los mediadores, donde se incluyen el apoyo social y los recursos de afrontamiento. Estos últimos son descritos como "los esfuerzos cognitivos y conductuales constantemente cambiantes que se desarrollan para manejar las demandas específicas, externas y/o internas, que son evaluadas como excedentes o desbordantes de los recursos del individuo" (Lazarus y Folkman, 1986: 164).

El concepto de afrontamiento ha sido desarrollado desde dos perspectivas teóricas y metodológicas (Sandín, 1995): la perspectiva disposicional y la situacional. La primera de ellas lo entiende como una predisposición o estilo personal, similar a un rasgo de personalidad, relativamente estable de afrontar el estrés. Desde la segunda perspectiva, la situacional, se considera que este viene determinado tanto por el contexto como por el propio sujeto, siendo, por tanto, más modificable. Se habla así de estilos y estrategias de afrontamiento, respectivamente (Vallejo-Sánchez y Pérez-García, 2015).

Dentro de las clasificaciones que permiten agrupar estas estrategias, Tobin et al. (1989) han desarrollado una de las más aceptadas. Estos autores distinguen entre las estrategias dirigidas al problema y las dirigidas hacia la emoción. Las primeras se orientan a definir el problema y buscar posibles soluciones al mismo. Las segundas se dirigen a controlar la respuesta emocional del individuo ocasionada por el problema. Se ha constatado la efectividad de las estrategias centradas en el problema (Gottlieb y Wolfe, 2002), aunque la disfuncionalidad de las estrategias centradas en la emoción ha sido más cuestionada (lavarone et al., 2014). Desde la perspectiva del tipo de respuesta, se ha elaborado otra clasificación que distingue entre el afrontamiento activo o de compromiso (engagement) y afrontamiento pasivo o de evitación (disengagement), según el sujeto haga intentos activos por manejar la situación o las emociones o se distancie del estresor o de las emociones derivadas del mismo (Carver y Connor-Smith, 2010; Vallejo-Sánchez y Pérez-García, 2015). Tradicionalmente, las estrategias de afrontamiento activo han sido descritas como adaptativas, mientras que las pasivas han sido consideradas como desadaptativas (Lazarus y Folkman, 1986). García-Alberca et al. (2012) han encontrado que las estrategias de evitación predicen mayores niveles de ansiedad en cuidadoras de personas con alzhéimer. Artaso et al. (2003) identifican la huida-evitación como predictora de sobrecarga, mientras que el apoyo social, estrategia de tipo activo, predice una reducción de la misma.

Sin embargo, la evidencia empírica tampoco ofrece, en este caso, resultados plenamente consistentes. Así, la investigación de Raggi et al. (2015) constata una significativa correlación con la carga del cuidador de ambos tipos de estrategias. Muela et al. (2002), a partir de una metodología propia que clasifica las estrategias en adecuadas e inadecuadas, encuentran una relación entre el nivel de carga que manifiestan las cuidadoras de personas con alzhéimer con el número de estrategias inadecuadas utilizadas. No obstante, no encuentran relación al analizar el número de estrategias adecuadas.

La aparente falta de consistencia de estos resultados ha llevado a muchos autores a constatar que lo realmente importante y adaptativo es la flexibilidad del afrontamiento; es decir, la capacidad del sujeto para 
utilizar la estrategia más apropiada en función de las demandas del contexto (Muela et al., 2002; VallejoSánchez y Pérez-García, 2015). Parece, por tanto, más conveniente abogar por una concordancia entre la situación y el tipo de estrategia, que por la utilización de un determinado estilo que predisponga al sujeto a utilizar los mismos tipos de estrategias con independencia de la situación en la que se apliquen.

Autores como Cerquera y Galvis (2014) o Raggi et al. (2015) apuntan a la necesidad de analizar también la influencia de variables sociodemográficas y/o relativas al entorno como mediadoras entre las estrategias de afrontamiento y los niveles de carga. En esta línea, es importante resaltar que el impacto del cuidado, así como la respuesta adaptativa de las cuidadoras, presenta una realidad diferente en el entorno rural frente al medio urbano. El contexto rural se caracteriza por una menor cantidad de servicios socionitarios así como dificultades de transporte (especialmente en invierno) y aislamiento (Dunn et al., 2016), pero también ofrece unas relaciones de proximidad más fuertes, fruto de una mayor cultura familiarista y comunitaria aún persistente hoy en día (Durán, 2011; Ehrlich et al., 2015; Lorenzo et al., 2014; Manso et al., 2013).

El objetivo del presente estudio es describir las estrategias de afrontamiento más utilizadas por las cuidadoras informales de personas con alzhéimer e identificar si se presentan diferencias en la utilización de estas estrategias en función de variables sociodemográficas, familiares y/o relativas a la tarea del cuidado y a las características del enfermo (consideradas como clínicas por parte de algunos autores).

\section{Método}

\section{- Diseño}

Se planteó un diseño transversal ex-post facto a través de la técnica de encuesta. La investigación se desarrolló en el ámbito de una asociación de ámbito rural de familiares de personas con alzhéimer de la comunidad autónoma de Castilla y León (España).

\section{- Participantes}

La encuesta fue dirigida a las cuidadoras informales de personas con alzhéimer de la asociación, utilizándose como único criterio de inclusión que estas llevaran ejerciendo la tarea del cuidado por un tiempo igual o superior a 3 meses. Del total de 89 cuidadoras registradas en la asociación en el momento de la recogida de datos, 84 cumplían el criterio de inclusión. Se obtuvieron un total de 57 respuestas, lo que supone una tasa de participación del $68 \%$.

El análisis del perfil de las personas encuestadas muestra que, según el género, se produce un claro predominio de las mujeres (84,2\%), quienes, además, presentan una edad media ligeramente inferior, 62,3 años frente a los 66 años del grupo de los hombres. La mayor parte de las cuidadoras están casadas o son pareja de hecho ( $86 \%$ ). Un $89,5 \%$ del total no trabaja en el momento de la recogida de datos y, en cuanto al nivel de estudios, se aprecia un amplio y similar porcentaje entre el grupo de sujetos que no tienen estudios $(42,1 \%)$ y los que solo tienen estudios primarios $(45,6 \%)$. 
Tabla 1. Información relacionada con las características de los participantes (porcentajes y medias)

\begin{tabular}{|l|c|c|}
\hline Variables & Categorías & Resultados \\
\hline \multirow{2}{*}{ Sexo } & Hombre & $15,8 \%$ \\
\hline \multirow{2}{*}{ Edad } & Mujer & $84,2 \%$ \\
\hline \multirow{2}{*}{ Estado civil } & Hombre & Media: 66 \\
\cline { 2 - 3 } & Mujer & Media: 62,3 \\
\hline \multirow{2}{*}{ Situación laboral } & Soltero & $1,8 \%$ \\
\hline \multirow{3}{*}{ Nivel de estudios } & Casado - Pareja de hecho & $86 \%$ \\
\cline { 2 - 3 } & Viudo & $12,2 \%$ \\
\cline { 2 - 3 } & Trabaja & $10,5 \%$ \\
\hline \multirow{2}{*}{$\mathbf{N}=\mathbf{5 7}$} & No trabaja & $89,5 \%$ \\
\cline { 2 - 3 } & Sin estudios & $42,1 \%$ \\
\cline { 2 - 3 } & Primarios & $45,6 \%$ \\
\cline { 2 - 3 } & Secundarios & $8,8 \%$ \\
\hline
\end{tabular}

Fuente: elaboración propia.

\section{- Procedimiento}

Inicialmente se realizó una solicitud al equipo directivo de la asociación para poder realizar la investigación. En febrero de 2016, este equipo autorizó la realización de la misma. Entre los meses de mayo y junio de ese mismo año se llevó a cabo el estudio de campo.

En cuanto a los aspectos éticos, señalar que se informó, por escrito, a todas las cuidadoras de los objetivos de la investigación, así como de las garantías de confidencialidad y anonimato de los datos ofrecidos, respetando así el artículo 24 de la Declaración de Helsinki. Todas las encuestadas dieron su consentimiento para la realización del estudio. En la mayor parte de los casos, fueron los profesionales de la asociación los encargados de facilitar los cuestionarios y el citado documento a las cuidadoras, quienes lo cumplimentaron. En aquellas situaciones en las que estos profesionales valoraron que era preferible que el cuestionario fuera aplicado mediante entrevista (debido especialmente a la elevada edad de alguna de las cuidadoras), se realizó de esta forma.

Los profesionales también se encargaron de solucionar posibles dudas que pudieran surgir. Todos estos aspectos habían sido previamente acordados entre la dirección de la asociación y el equipo de investigación.

\section{- Instrumento}

Para la recogida de datos se han utilizado los siguientes instrumentos: 
- Inventario de Estrategias de Afrontamiento (Tobin et al., 1989): Se ha utilizado la adaptación española de Cano et al. (2007). La escala original presenta una estructura jerárquica compuesta por ocho estrategias primarias, cuatro secundarias y dos terciarias que surgen respectivamente de la agrupación empírica de las anteriores. La adaptación española reduce el número inicial de ítems de 72 a 40. La escala resultante ofrece buenas propiedades psicométricas con coeficientes de consistencia interna entre 0,63 y 0,89 y una adecuada validez convergente. Estos autores obtuvieron en el proceso de validación una estructura factorial de primer orden coincidente con la escala original; sin embargo, no se confirmaron las estructuras de segundo y tercer orden originales. Estas no serán, por tanto, utilizadas como referencia dentro del análisis de la presente investigación.

Las ocho escalas primarias son (Cano et al., 2007):

- La resolución de problemas: estrategia cognitiva y conductual dirigida a eliminar el estrés modificando la situación que lo produce.

- La reestructuración cognitiva: estrategia cognitiva que modifica el significado de la situación estresante.

- El apoyo social: estrategia referida a la búsqueda de apoyo emocional.

- La expresión emocional: estrategia encaminada a liberar las emociones que acontecen en el proceso de estrés.

- La evitación de problemas: estrategia que incluye la negación y evitación de pensamientos o actos relacionados con el acontecimiento estresante.

- El pensamiento desiderativo: estrategia cognitiva que refleja el deseo de que la realidad no fuera estresante.

- La retirada social: estrategia de retirada de amigos, familiares, compañeros y personas significativas asociada con la reacción emocional en el proceso estresante.

- La autocrítica: estrategia basada en la autoinculpación y la autocrítica por la ocurrencia de la situación estresante o su inadecuado manejo².

- Cuestionario ad hoc: Se diseñó un cuestionario propio con el objetivo de recoger la información relativa a la familia y al entorno y la relativa al cuidado y a la persona con alzhéimer. La tabla 2 presenta las variables y categorías incorporadas en este cuestionario. No se han utilizado variables tipo escala, por lo que no se han realizado análisis psicométricos del mismo.

\section{- Análisis de datos}

Los resultados de las variables relativas al entorno familiar y las relativas al cuidado y a las características de la persona con alzhéimer se presentan mediante porcentajes. Para el grado de utilización de las estrategias de afrontamiento se han descrito las puntuaciones medias, desviaciones típicas y cuartiles.

Se empleó la prueba de Kolmogorov-Smirnov para comprobar la hipótesis de normalidad de las variables cuantitativas. Dado que los resultados de la misma no permitieron asumir un comportamiento de normalidad

2. Se remite al lector a la obra de Cano et al. (2007) para una lectura de los enunciados que describen cada dimensión. 
de las variables y teniendo en cuenta el reducido tamaño de la muestra, se utilizaron, para el análisis de las diferencias entre grupos, técnicas no paramétricas.

De forma específica, se utilizó la prueba $U$ de Mann Whitney, cuando se trataba de analizar las diferencias entre dos grupos, y la prueba $\mathrm{H}$ de Kruskal Wallis, cuando el objetivo era describir diferencias entre más de dos grupos. Se han considerado como significativos los valores de $p<0,01$ y $p<0,05$, aunque, en algunos casos, se hará referencia a aquellos con un $\mathrm{p}<0,1$.

\section{Resultados}

\section{- Variables familiares, relativas al cuidado y a la persona con alzhéimer}

La tabla 2 recoge la información relativa a las variables contextuales (familiares y relacionales), las relativas a la tarea del cuidado y las relativas a las características de la persona con alzhéimer y su nivel de deterioro. Se produce un claro predominio de personas con alzhéimer que viven solo con su cónyuge o, en su caso, con su cónyuge y alguno de los hijos. Ambos casos presentan porcentajes del $35,1 \%$. Un $38,6 \%$ de los sujetos son esposas/os de la persona con alzhéimer. El mismo porcentaje se observa para el caso de las hijas/os.

En cuanto al apoyo social, la tabla muestra que más de un tercio de los sujetos $(36,8 \%)$ establece relaciones sociales con una frecuencia diaria. El $24,6 \%$ se relaciona con gente del entorno varias veces a la semana.

Un $45,6 \%$ de los sujetos consideran que la tarea del cuidado puede haber afectado a las relaciones familiares.

El segundo bloque describe las características de la tarea del cuidado desde la perspectiva de las cuidadoras. La mayoría (93\%) han tenido ya alguna experiencia anterior de cuidado. Refiriéndose ya a la tarea del cuidado por la que han sido encuestadas, más de la mitad $(56,1 \%)$ llevan realizando esta tarea por un periodo superior a los tres años. Un $28,1 \%$ llevan cuidando a su familiar por un periodo inferior al año.

Un 43,9\% de las cuidadoras señalan que no han recibido información sobre la tarea del cuidado.

El tercer bloque de variables describe la situación de la persona con alzhéimer. Un 43,8\% tiene más de 80 años y, dentro de este grupo, casi un $40 \%$ supera los 85 años (un $17,5 \%$ de la muestra total). En cuanto al género, la muestra está bastante equilibrada con un ligero predominio del género masculino $(52,6 \%)$. Se ha estudiado también, en cuanto variable clínica, el nivel de deterioro. Los resultados reflejan que un 49,1\% presentan un deterioro moderado y un $36,8 \%$ un grave deterioro. 


\begin{tabular}{|c|c|c|}
\hline Variables & Categorías & Porcentaje \\
\hline \multicolumn{3}{|c|}{ Variables relativas al ámbito familiar y relacional } \\
\hline \multirow{5}{*}{ Composición familiar } & Sólo cónyuge & $35,1 \%$ \\
\hline & Cónyuge e hijos & $35,1 \%$ \\
\hline & Sólo hijos & $15,8 \%$ \\
\hline & Cónyuge y otros familiares (sin hijos) & $8,8 \%$ \\
\hline & Hijos y otros familiares (sin cónyuge) & $5,3 \%$ \\
\hline \multirow{4}{*}{ Relación de parentesco } & Esposo/a & $38,6 \%$ \\
\hline & $\mathrm{Hijo} / \mathrm{a}$ & $38,6 \%$ \\
\hline & Nuera/yerno & $15,8 \%$ \\
\hline & Otros & $7 \%$ \\
\hline \multirow{5}{*}{ Frecuencia de las relaciones sociales } & Todos los días & $36,8 \%$ \\
\hline & Varias veces a la semana & $24,6 \%$ \\
\hline & Una vez a la semana & $12,3 \%$ \\
\hline & Varias veces al mes & $10,5 \%$ \\
\hline & Una vez al mes o menos & $15,8 \%$ \\
\hline \multirow{2}{*}{ ¿Afectada relación familiar? } & Sí & $45,6 \%$ \\
\hline & No & $54,4 \%$ \\
\hline \multicolumn{3}{|l|}{ Variables relativas al cuidado } \\
\hline \multirow{2}{*}{ ¿Ha cuidado anteriormente? } & Sí & $93 \%$ \\
\hline & No & $7 \%$ \\
\hline \multirow{3}{*}{ Tiempo que lleva cuidando } & Un año o menos & $28,1 \%$ \\
\hline & Entre 1 y 3 años & $15,8 \%$ \\
\hline & Más de 3 años & $56,1 \%$ \\
\hline \multirow{2}{*}{ ¿Ha recibido información? } & Sí & $56,1 \%$ \\
\hline & No & $43,9 \%$ \\
\hline \multicolumn{3}{|c|}{ Variables relativas a la persona con alzhéimer } \\
\hline \multirow{4}{*}{ Edad } & 75 años o menos & $31,6 \%$ \\
\hline & De 76 a 80 años & $24,6 \%$ \\
\hline & De 81 a 85 años & $26,3 \%$ \\
\hline & 86 o más años & $17,5 \%$ \\
\hline \multirow{2}{*}{ Sexo } & Hombre & $52,6 \%$ \\
\hline & Mujer & $47,4 \%$ \\
\hline \multirow{3}{*}{ Nivel de deterioro } & Leve & $14 \%$ \\
\hline & Moderado & $49,1 \%$ \\
\hline & Grave & $36,8 \%$ \\
\hline
\end{tabular}

Fuente: elaboración propia. 


\section{- Estrategias de afrontamiento utilizadas}

La tabla 3 describe las puntuaciones relativas a la utilización de las distintas estrategias. Estas han sido organizadas de acuerdo a los dos sistemas de clasificación anteriormente presentados: centradas en el problema y centradas en la emoción, por un lado, y activas y pasivas, por otro. Las puntuaciones más altas aparecen en las cuatro estrategias de tipo activo que han sido tradicionalmente consideradas como adaptativas: la resolución de problemas (media de 21,9), el apoyo social (media de 18,1), la expresión emocional (media de 16,9) y la reestructuración cognitiva (media de 16,7). No obstante, una estrategia considerada como inadaptativa, el pensamiento desiderativo, obtiene también una alta puntuación media (19). La dispersión en las respuestas, medida a través de la desviación típica, es mayor, en líneas generales, en las estrategias desadaptativas.

\begin{tabular}{|c|c|c|c|c|c|c|}
\hline \multicolumn{2}{|l|}{ Tipo } & Estrategia de afrontamiento & M (D.T.) & Q1 & Q2 & Q3 \\
\hline \multirow{2}{*}{ Centradas en el problema } & \multirow{4}{*}{ Activas } & Resolución de problemas (REP) & $21,9(3,7)$ & 18,5 & 24 & 25 \\
\hline & & Reestructuración cognitiva (REC) & $16,7(4,6)$ & 13 & 16,5 & 21 \\
\hline \multirow{2}{*}{ Centradas en la emoción } & & Apoyo social (APS) & $18,1(4,6)$ & 14 & 18 & 21 \\
\hline & & Expresión emocional (EEM) & $16,9(5)$ & 13,2 & 17 & 21 \\
\hline \multirow{2}{*}{ Centradas en el problema } & \multirow{4}{*}{ Pasivas } & Evitación de problemas (EVP) & $12,8(4,7)$ & 9 & 13 & 16 \\
\hline & & Pensamiento desiderativo (PSD) & $19(5,3)$ & 15,5 & 20 & 25 \\
\hline \multirow{2}{*}{ Centradas en la emoción } & & Retirada social (RES) & $13,7(5)$ & 10,5 & 13 & 17 \\
\hline & & Autocrítica (AUC) & $9,3(4,8)$ & 5 & 8,5 & 11 \\
\hline
\end{tabular}

Fuente: elaboración propia.

\section{- Diferencias en función de las variables estudiadas}

Para explorar las diferencias en la utilización de las estrategias de afrontamiento según las variables estudiadas, se ha procedido a realizar un análisis no paramétrico cuyos resultados, agrupados por bloques, se muestran en la tabla 4.

Por lo que se refiere a las variables demográficas, únicamente se encuentran diferencias estadísticamente significativas según el nivel de estudios en la estrategia de retirada social $(p=0,031)$, utilizando en menor medida esta estrategia las cuidadoras que poseen estudios secundarios o universitarios (ver Anexo 1 para una descripción de las puntuaciones medias en las distintas categorías de respuesta). Trabajando a un nivel de significación $p<0,1$, se presentan diferencias entre hombres y mujeres cuidadoras en la estrategia de apoyo social $(p=0,052)$ y, en función de la edad, en las estrategias de resolución de problemas $(p=0,058)$ y evitación de problemas $(p=0,053)$.

Es en el bloque de variables relativas al contexto familiar y relacional donde aparecen un mayor número de diferencias significativas. Así, atendiendo a la variable relación de parentesco, aparecen diferencias en 
la estrategia de autocrítica $(p=0,042)$, siendo esta utilizada en menor medida por los esposos o hijos de la persona con alzhéimer que por otros familiares. La distinta frecuencia de las relaciones sociales establece diferencias en las estrategias de apoyo social $(p=0,047)$ y expresión emocional $(p=0,030)$; y, variando el nivel de significación, en el pensamiento desiderativo $(p=0,80)$. En este último caso, la mayor frecuencia de las relaciones está asociada a un incremento en la utilización de la misma.

El hecho de que la tarea de cuidado haya o no afectado a las relaciones familiares va asociado a una utilización significativamente diferente en cuatro de las ocho estrategias primarias analizadas (una de ellas a un nivel de significación $p<0,1)$ : expresión emocional $(p=0,015)$, pensamiento desiderativo $(p=0,003)$, apoyo social $(p=0,002)$ y resolución de problemas $(p=0,096)$. En todos los casos se produce una mayor utilización de la estrategia mencionada por parte de las cuidadoras que manifiestan una afectación en las relaciones familiares.

\begin{tabular}{|c|c|c|c|c|c|c|c|c|}
\hline & REP & REC & APS & EEM & EVP & PSD & RES & AUT \\
\hline \multicolumn{9}{|l|}{ Variables personales } \\
\hline Sexo $^{\mathrm{a}}$ & 0,179 & 0,404 & 0,052 & 0,120 & 0,382 & 0,169 & 0,947 & 0,943 \\
\hline Edad $^{\mathrm{b}}$ & 0,058 & 0,450 & 0,602 & 0,844 & 0,053 & 0,834 & 0,778 & 0,770 \\
\hline Situación laborala & 0,679 & 0,625 & 0,802 & 0,841 & 0,097 & 0,865 & 0,713 & 0,347 \\
\hline Nivel de estudios $^{b}$ & 0,822 & 0,526 & 0,619 & 0,261 & 0,232 & 0,142 & 0,031 & 0,390 \\
\hline \multicolumn{9}{|c|}{ Variables relativas al ámbito familiar } \\
\hline Composición familiar ${ }^{b}$ & 0,700 & 0,245 & 0,780 & 0,916 & 0,772 & 0,088 & 0,255 & 0,213 \\
\hline Parentesco ${ }^{b}$ & 0,220 & 0,441 & 0,416 & 0,821 & 0,497 & 0,968 & 0,143 & 0,042 \\
\hline $\begin{array}{l}\text { Frecuencia relaciones } \\
\text { sociales }^{\mathrm{b}}\end{array}$ & 0,342 & 0,158 & 0,047 & 0,030 & 0,864 & 0,080 & 0,615 & 0,225 \\
\hline $\begin{array}{l}\text { Afectadas relaciones } \\
\text { familiares }^{\mathrm{a}}\end{array}$ & 0,096 & 0,607 & 0,002 & 0,015 & 0,908 & 0,003 & 0,380 & 0,261 \\
\hline \multicolumn{9}{|c|}{ Variables relativas a la tarea del cuidado } \\
\hline Cuidado anteriormente $^{\mathrm{a}}$ & 0,697 & 0,370 & 0,312 & 0,109 & 0,677 & 0,448 & 0,019 & 0,374 \\
\hline Tiempo cuidado ${ }^{b}$ & 0,744 & 0,510 & 0,774 & 0,588 & 0,091 & 0,004 & 0,425 & 0,150 \\
\hline Recibido información ${ }^{a}$ & 0,144 & 0,696 & 0,149 & 0,098 & 0,053 & 0,433 & 0,312 & 0,377 \\
\hline \multicolumn{9}{|c|}{ Variables relativas a la persona con alzhéimer } \\
\hline $\operatorname{Sexo}^{\mathrm{a}}$ & 0,359 & 0,818 & 0,044 & 0,999 & 0,674 & 0,927 & 0,013 & 0,522 \\
\hline Edad $^{\mathrm{b}}$ & 0,421 & 0,723 & 0,579 & 0,818 & 0,359 & 0,421 & 0,516 & 0,082 \\
\hline Nivel de deterioro ${ }^{b}$ & 0,217 & 0,806 & 0,154 & 0,031 & 0,765 & 0,176 & 0,521 & 0,220 \\
\hline
\end{tabular}

a. Prueba U de Mann Whitney.

b. Prueba $\mathrm{H}$ de Kruskal Wallis.

Fuente: elaboración propia. 
El haber tenido experiencia anterior de cuidado guarda relación con la utilización de la retirada social $(p=0,019)$. Esta estrategia es utilizada, en mayor medida, por aquellas cuidadoras con esta experiencia. La variable tiempo de cuidado produce diferencias en el pensamiento desiderativo $(p=0,004)$ y evitación de problemas $(p=0,091)$. Finalmente, atendiendo a la variable información relativa al cuidado, aparecen diferencias $(p<0,1)$ en la estrategia de evitación de problemas $(p=0,053)$.

Dentro del último bloque, relativo a las características demográficas y clínicas de la persona con alzhéimer se observan diferencias en las estrategias apoyo social $(p=0,044)$ y retirada social $(p=0,013)$ según el sexo. En ambos casos se produce una mayor utilización de las estrategias cuando la persona con alzhéimer es varón. La edad sólo genera diferencias, trabajando a un nivel $p<0,1$, en la estrategia de autocrítica $(p=0,082)$. La expresión emocional $(p=0,031)$ es la única estrategia en la que se observan diferencias según la variable clínica nivel de deterioro, estando su mayor utilización asociada a mayores niveles de deterioro.

\section{Discusión y conclusiones}

El presente estudio se ha centrado en el análisis de las estrategias de afrontamiento utilizadas por un grupo de cuidadoras de personas con alzhéimer y en los factores personales, relativos al entorno familiar y al contexto del cuidado que guardan relación con las mismas.

El perfil descriptivo de la muestra ofrece, en cuanto a la distribución por género, unos resultados coincidentes con investigaciones previas, tanto en el contexto nacional como internacional (Bielsa, 2013; Manso et al., 2013; Rodríguez-González et al., 2017; Wennberg et al., 2015). Las tareas de cuidado siguen estando determinadas por razones de género (Coira y Bailon, 2014). Así, la población femenina experimenta mayores dificultades para estructurar sus vidas y una mayor tensión emocional al tener que solapar tiempos de dimensiones diferentes. Por un lado, los tiempos dedicados a la productividad y a la rentabilidad y, por otro, los tiempos dedicados al cuidado (Carrasco, 2016; Carrasco y Recio, 2014).

La presente investigación ha puesto de manifiesto una mayor utilización de las estrategias activas, tanto centradas en el problema como centradas en la emoción. Este resultado, consistente con los obtenidos en la mayoría de las investigaciones (Artaso et al., 2003; García et al., 2016; García-Alberca et al., 2012; Lazarus y Folkman, 1986), revela una mayor utilidad adaptativa de estas en la reducción del estrés. lavarone et al. (2014) señalan, al respecto, que las cuidadoras de personas con alzhéimer tienen más tendencia a "ir hacia" al paciente, tanto en el sentido conductual como en el emocional.

Observando de forma específica las distintas estrategias, se ha encontrado una mayor utilización de la resolución de problemas y la reestructuración cognitiva, por un lado, y el apoyo social y la expresión emocional, por otro. La elevada utilización de la primera de las estrategias mencionadas es consistente con lo encontrado por Cerquera y Galvis (2014), utilizando, en este caso, la Escala de Estrategias de Coping - Modificada (EEC-M). 
El apoyo social es otra estrategia muy utilizada. Se trata de una estrategia efectiva en la reducción del estrés (Artaso et al., 2003; Coira y Bailon, 2014; García et al., 2016, Moreno-Cámara et al., 2016; Rodríguez-Madrid et al., 2019). Ruiz de Alegría et al. (2006) señalan que las personas que cuidan a personas con alzhéimer manifiestan la necesidad de expresar y compartir sus sentimientos de angustia, tristeza o culpa ante personas cercanas, por lo que necesitan hacer un alto uso de esta estrategia. La investigación de Ehrlich et al. (2015) refleja, además, un mayor uso de esta estrategia en las cuidadoras en entornos rurales caracterizados por una mayor cultura familiarista y comunitaria.

Conviene advertir, no obstante, que la investigación de Raggi et al. (2015) muestra una alta relación entre elevados niveles de angustia en cuidadoras de personas con alzhéimer y una mayor frecuencia de utilización del apoyo social. Esta realidad pone de manifiesto la importancia de la flexibilidad, es decir de la utilización de las estrategias en función de factores personales o situacionales (Muela et al., 2002; Vallejo-Sánchez y Pérez-García, 2015).

La flexibilidad y adaptación de las estrategias al contexto puede ser la explicación de la elevada utilización que las personas encuestadas hacen de una de las estrategias consideradas como desadaptativas: el pensamiento desiderativo. Otras estrategias desadaptativas como son la autocrítica y la evitación de problemas son escasamente utilizadas. Artaso et al. (2003) identifican esta última como importante predictora de la sobrecarga.

El análisis de las diferencias en la utilización de estas estrategias en función de las variables personales de la cuidadora, familiares, situacionales, relativas al cuidado y relativas a las características de la persona con alzhéimer ha permitido revelar varios hechos significativos. Así, se produce una mayor utilización por parte las mujeres de la estrategia centrada en la emoción: el apoyo social. Resultados que reflejan una mayor frecuencia en el uso de estrategias centradas en la emoción por parte de mujeres cuidadoras de personas con alzhéimer han sido encontrados por lavarone et al. (2014). Se plantea la hipótesis de que estos resultados pueden ser explicados en función de los roles sociales asignados a las mujeres. A estas se les exige, aún hoy en día, seguir manteniendo el rol de 'personas que cuidan' (Coira y Bailon, 2014; Manso et al., 2013), lo que produce un mayor agotamiento de los recursos físicos y psicológicos. Esto hace que sea más necesaria la utilización de este elemento modulador de la carga emocional.

El análisis en función del género ha revelado, sin embargo, algunos resultados discordantes. Así, la reciente investigación de Lozano et al. (2017) muestra un mayor uso de la estrategia de apoyo social por parte de los hombres. Además, otras variables como son el nivel socioeconómico (Howerton y Van Gundy, 2009) o las expectativas del cuidador (Pinquart y Sörensen, 2006) condicionan la influencia de la misma. Finalmente, el reciente análisis de Rodríguez-Madrid et al. (2019) revela que las redes sociales de apoyo son más amplias en el caso de los hombres.

La variable edad guarda relación con dos estrategias centradas en el problema: la resolución de problemas y la evitación de problemas. El análisis de las puntuaciones de esta variable muestra que las respuestas más desadaptativas (menores puntuaciones en resolución de problemas y mayores en evitación de problemas) se sitúan en el grupo de cuidadoras entre 56 y 65 años; es decir, normalmente hijas/os en edades ya avanzadas, próximas a la jubilación. Probablemente se trata de la etapa más delicada, ya que la cuidadora se enfrenta a un incremento en la intensidad de la tarea del cuidado en un momento en el que aún debe 
compatibilizar esta con su rol laboral. Son escasos los estudios que analizan la influencia de esta variable en cuidadoras de personas con alzhéimer. Sin embargo, se ha encontrado una influencia significativa en otros colectivos, como padres de niños con trastorno del espectro autista (Luque et al., 2017).

Los datos revelan una importante y negativa relación entre el nivel de estudios y una de las estrategias consideradas como desadaptativas: la retirada social. Artaso et al. (2002) han encontrado resultados similares. Un mayor nivel académico, no solo puede permitir un mayor conocimiento y, por tanto, una mayor utilización de los recursos del entorno, sino que se presenta generalmente unido a unas mejores condiciones laborales y salariales. Entre otros aspectos, esto reduce en la familia el impacto de la contratación de ayuda remunerada (Durán, 2018; Ruiz-Adame et al., 2019).

En un segundo grupo de variables, se incluyen aquellas relativas al contexto familiar y relacional. Los pensamientos de autoinculpación y autocrítica son significativamente menores en el caso de los parientes más próximos (cónyuges o hijos). Una mayor implicación en la tarea del cuidado y un mayor vínculo emocional con la persona con alzhéimer pueden ser elementos que expliquen este resultado. No obstante, los resultados de las investigaciones acerca de la influencia de la relación de parentesco sobre la carga no han sido consistentes. Así, mientras que el estudio de Rodríguez-González et al. (2017) revela una relación inversa entre el grado de parentesco y la carga, Raggi et al. (2015) constatan mayores índices de estrés en cuidadoras que viven junto al paciente, especialmente en el caso de los cónyuges.

Uno de los resultados más relevantes es la relación que se establece entre el hecho de que se hayan visto afectadas las relaciones familiares y las dos estrategias activas centradas en la emoción (expresión emocional y apoyo social). El grupo que manifiesta una mayor repercusión de la tarea del cuidado en las relaciones familiares utiliza con mayor frecuencia estas estrategias adaptativas de tipo emocional. Se plantea la hipótesis de que el sufrimiento añadido que puede provocar el deterioro de las relaciones familiares lleva a las cuidadoras a gestionar esta situación, en mayor medida, en el plano emocional. Apoyando esta idea, se observa en este grupo una menor incidencia de estrategias centradas en el problema, como son el pensamiento desiderativo o la resolución de problemas. Conviene poner de manifiesto esta relación, ya que la mayor incidencia de las disfunciones familiares aparece en varias investigaciones asociadas a la situación de cuidado (López et al., 2009; Ory et al., 1999).

Finalmente se han incluido variables relativas al estresor, concretamente las características del cuidado y las relativas a la persona con alzhéimer. La necesidad de inclusión de estas variables en el análisis es puesta de relieve por autores como Montorio et al. (1999), quienes señalan que la eficacia de las estrategias de afrontamiento empleadas por las cuidadoras de personas con alzhéimer dependerá de la concordancia entre la naturaleza del estresor y el tipo de estrategia utilizada. La presente investigación ha revelado relaciones significativas entre estas variables y algunas estrategias de tipo pasivo o desadaptativas.

La estrategia de retirada social es utilizada, en mayor medida, por las cuidadoras que han tenido experiencia anterior de cuidado. Se ha encontrado también una significativa relación entre el tiempo de cuidado y las dos estrategias de tipo pasivo centradas en el problema, como son la evitación de problemas y el pensamiento desiderativo. Autores como Ruiz et al. (2016) encuentran, igualmente, una relación entre el mayor tiempo de cuidado y el afrontamiento pasivo. Sin embargo, en el presente estudio, el análisis del sentido de esta relación revela varios hechos significativos. En primer lugar, en ninguna de las dos variables se aprecia una 
relación lineal con el mayor o menor tiempo de cuidado. En segundo lugar, se observa que el patrón de relación es distinto en ambos casos. Así, mientras que el uso del pensamiento desiderativo decrece en los periodos intermedios de cuidado (entre 1 y 3 años); en el caso de la estrategia de evitación de problemas, su uso es más acentuado en este periodo de tiempo. Sin duda, estos resultados apuntan de nuevo a la idea de la flexibilidad en la utilización de las estrategias de afrontamiento (Vallejo-Sánchez y Pérez-García, 2015), ya que el mayor o menor uso de cada una de ellas puede ser el resultado de los ajustes adaptativos que hace el sujeto después de estar un tiempo al cuidado de la persona con alzhéimer.

Son escasos los estudios que analizan en qué medida las características de la persona con alzhéimer pueden producir diferencias en la utilización de las distintas estrategias. El mayor uso del apoyo social en cuidadoras de varones con alzhéimer refuerza un resultado anterior: esta estrategia activa centrada en la emoción es más utilizada por las mujeres (lavarone et al., 2014). Se asume, para ello, el hecho de que muchas de estas cuidadoras son cónyuges del paciente y, por tanto, mujeres. Manteniendo este argumento, se observa que en este grupo (cuidadoras de varones con alzhéimer) se produce una mayor y significativa utilización de otra estrategia de tipo emocional, aunque en este caso pasiva, como es la retirada social.

Finalmente, considerando la variable clínica nivel de deterioro, la investigación ha revelado un mayor uso de la estrategia de expresión emocional en cuidadoras que atienden a personas con un elevado nivel de deterioro. Resultados similares han sido encontrados en estudios como el de lavarone et al. (2014). La necesidad de expresar emociones y sentimientos se presenta, de nuevo, vinculada a aquellas situaciones que generan una mayor demanda de cuidados.

A modo de conclusión, señalar que la flexibilidad del afrontamiento ha sido una de las perspectivas teóricas más asumidas en los últimos años. El presente estudio aporta un nuevo apoyo empírico a este enfoque. La capacidad para responder de forma adaptativa en función de las distintas realidades parece ser un elemento clave en la utilización eficaz del afrontamiento.

Los resultados revelan la necesidad de formar en el manejo flexible y adaptativo de estas estrategias en función de los rasgos personales y las demandas específicas del contexto. Igualmente, es importante desarrollar medidas sociosanitarias que prevengan y/o amortigüen la sobrecarga y, en este sentido, el acceso a redes de apoyo social se presenta como una de las más eficaces, al tratarse además de una de las grandes fortalezas del medio rural.

No debe olvidarse la distribución desigual que persiste en la responsabilidad del cuidado entre hombres y mujeres. Las medidas dirigidas a este colectivo no deberán descuidar este elemento, favoreciendo un reparto más equitativo de estas responsabilidades y estableciendo medidas que favorezcan la conciliación de la vida familiar y laboral. Todo ello en un entorno, como el rural, donde la feminización de esta tarea es, como se ha señalado, más persistente aún.

En cuanto a las limitaciones del presente estudio, señalar que es necesario profundizar en esta línea de investigación con muestras mayores y análisis multivariantes que permitan conocer la posible interacción entre las variables estudiadas y las diferentes estrategias utilizadas. Estas técnicas permitirán introducir mecanismos de control en el análisis de los datos que, además de favorecer el establecimiento de posibles relaciones causa-efecto, ayudarán a identificar factores predictores en la reducción de la sobrecarga. 
Adelman, R. et al. (2014): "Caregiver burden: a clinical review". The Journal of the American Medical Association, 311 (10): 1052-1060.

Alzheimer's Association (2015): “2015 Alzheimer's disease facts and figures”. Alzheimer’s \& Dementia, 11(3): 332-384.

Artaso, B. et al. (2002): "Estrategias de afrontamiento de las cuidadoras informales del paciente con demencia". Cuadernos de medicina psicosomática y psiquiatría de enlace, 60-61: 38-45.

Artaso, B. et al. (2003): "Cuidados informales en la demencia: predicción de sobrecarga en cuidadoras familiares". Revista Española de Geriatría y Gerontología, 38 (4): 212-218.

Bielsa, M. (2013): "La enfermedad de Alzheimer y el cuidador principal”. Nuberos Científica, 2(10): 6-12.

Bruvik, F. et al. (2012): "The quality of life of people with dementia and their family carers". Dementia and Geriatric Cognitive Disorders, 34 (1): 7-14.

Cano, F. et al. (2007): “Adaptación española del Inventario de Estrategias de Afrontamiento”. Actas Españolas de Psiquiatría, 35 (1): 29-39.

Carrasco, C. (2016): "Tiempos en conflicto, sociedades insostenibles, diálogos necesarios". Revista de Economía Crítica, 22: 108-125.

Carrasco, C. y Recio, A. (2014): “Del tiempo medido a los tiempos vividos”. Revista de economía crítica, 17: 82-97

Carver, C. y Connor-Smith, J. (2010): “Personality and coping”. Annual Review of Psychology, 61: 679-704.

Cerquera, A. y Galvis, M. (2014): "Efectos de cuidar personas con Alzheimer: un estudio sobre cuidadores formales e informales”. Pensamiento psicológico, 12 (1): 149-167.

Coira, G. y Bailon, M. (2014): “La invisibilidad de los cuidados que realizan las mujeres”. Atención Primaria, 46 (6): 271-272.

Daley, R. et al. (2017): "In this together' or 'Going it alone': Spousal dyad approaches to Alzheimer's". Journal of Aging Studies, 40: 57-63.

Dunn, D. J. et al. (2016): "Rural caregivers of persons with dementia: Review of the literature guided by Rogers' Science of Unitary Human Beings”. Visions: The Journal of Rogerian Nursing Science, 22 (1): 16-24.

Durán, M. A. (2011): “Las personas mayores en el medio rural y urbano”, en Instituto de Mayores y Servicios Sociales: Envejecimiento activo. Libro Blanco. Madrid: Ministerio de Sanidad, Política Social e Igualdad.

Durán, M. A. (2018): “Las cuentas del cuidado”. Revista española de control externo, 58 (20): 57-89.

Ehrlich, K. et al. (2015): "Family caregivers'assessments of caring for a relative with dementia: a comparison of urban and rural areas". International Journal of Older People Nursing, 10 (1): 27-37.

García, F. et al. (2016): "Bienestar psicológico, estrategias de afrontamiento y apoyo social en cuidadores informales". Psicoperspectivas, 15 (3): 87-97.

García-Alberca, J. M. et al. (2012): "Disengagement coping partially mediates the relationship between caregiver burden and anxiety and depression in caregivers of people with Alzheimer's disease. Results from the MÁLAGA-AD study". Journal of Affective Disorders, 136 (3): 848-856. 
Gottlieb, B. y Wolfe, J. (2002): "Coping with family caregiving to persons with dementia: A critical review". Aging \& Mental Health, 6 (4): 325-342.

Howerton, A. y Van Gundy, K. (2009): "Sex Differences in Coping Styles and Implications for Depressed Mood". International Journal of Stress Management, 16 (4): 333-350.

lavarone, A. et al. (2014): "Caregiver burden and coping strategies in caregivers of patients with Alzheimer's disease". Neuropsychiatric Disease and Treatment, 10: 1407-1413.

Lazarus, R. y Folkman, S. (1986): Estrés y procesos cognitivos, Barcelona: Martínez Roca.

López, M. et al. (2009): "El rol de cuidador de personas dependientes y sus repercusiones sobre su calidad de vida y su salud". Revista Clínica de Medicina de Familia, 2(7): 332-334.

Lorenzo, T. et al. (2014): "Caracterización de un colectivo de cuidadores informales de acuerdo a su percepción de la salud”. Aposta: Revista de ciencias sociales, (62): 1-20.

Lozano, M. et al. (2017): "Health-related quality of life in Spanish informal caregivers: gender differences and support received". Quality of Life Research, 26: 3227-3238.

Luque, B. et al. (2017): "The role of coping strategies and self-efficacy as predictors of life satisfaction in a sample of parents of children with autism spectrum disorder". Psicothema, 29 (1): 55-60.

Mahdavi, B. et al. (2017): "Effects of spiritual group therapy on caregiver strain in home caregivers of the elderly with Alzheimer's disease". Archives of Psychiatric Nursing, 31: 269-273.

Manso, M. E. et al. (2013): "Salud y sobrecarga percibida en personas cuidadoras familiares de una zona rural". Clínica y Salud, 24: 37-45.

Martínez-Marcos, M. y De la Cuesta-Benjumea, C. (2013): "How women caregivers deal with their own long-term illness: a qualitative study". Journal of Advanced Nursing, 70: 1825-1836.

McConaghy, R. y Caltabiano, M. (2005): “Caring for a person with dementia: Exploring relationships between perceived burden, depression, coping and well-being". Nursing \& Health Sciences, 7 (2): 81-91.

Montorio, I. et al. (1999): "El cuidado del anciano en el ámbito familiar”, en Izal, M. y Montorio, I. (eds.): Gerontología conductual. Bases para la intervención y ámbitos de aplicación. Madrid: Síntesis.

Moreno-Cámara, S. et al. (2016): "Problemas en el proceso de adaptación a los cambios en personas cuidadoras familiares de mayores con demencia”. Gaceta Saniaria, 30 (3): 201-207.

Muela, J.A. et al. (2002): "Comparación entre distintas clasificaciones de las estrategias de afrontamiento en cuidadores de enfermos de alzheimer". Psicothema, 14 (3): 558-563.

Organización Mundial de la Salud (2016): Demencia. Informe de la Secretaría. Consejo Ejecutivo. EB139/3. 139a reunión, 29 de abril de 2016 (en línea). <http://apps.who.int/iris/handle/10665/250901>, acceso 20 de Junio de 2017.

Ory M. et al. (1999): "Prevalence and impact of caregiving: a detailed comparison between dementia and non dementia caregivers". Gerontologist, 39 (2): 177-185.

Pearlin, L. I. et al. (1990): "Caregiving and the stress process: An overview of concepts and their measures". Gerontologist, 30 (5): 583-594.

Pinquart, M. y Sörensen, S. (2006): "Helping caregivers of persons with dementia: which interventions work and how large are their effects?". International Psychogeriatrics, 18: 577-595. 
Raggi, A. et al. (2015): "The burden of distress and related coping processes in family caregivers of patients with Alzheimer's disease living in the community". Journal of the Neurological Sciences, 358: 77-81.

Rodríguez-González, A. M. et al. (2017): "Estudio observacional transversal de la sobrecarga en cuidadoras informales y los determinantes relacionados con la atención a las personas dependientes”. Atención Primaria, 49 (3): 156-165.

Rodríguez-Madrid, N. et al. (2019): "Gender differences in social support received by informal caregivers: A personal network analysis approach". International Journal of Environmental Research and Public Health, 16: 91.

Rubio, M. et al. (2017): "Adaptando mi vida: vivencias de cuidadores familiares de personas con enfermedad de Alzheimer”. Gerokomos, 29: 54-58.

Ruiz, M. D. et al. (2016): "Evaluación de los estilos de afrontamiento utilizados en cuidadores familiares de enfermos crónicos complejos". European Journal of Health Research, 2 (3): 83-93.

Ruiz-Adame, M. et al. (2019): "The opportunity costs of caring for people with dementia in Southern Spain". Gaceta Sanitaria, $33(1): 17-23$.

Ruiz de Alegría, B. et al. (2006): "Estrategias de Afrontamiento beneficiosas para las mujeres que cuidan de un progenitor con Alzheimer". Index de Enfermería, 15 (54): 10-14.

Sandín, B. et al. (1995): "Trastornos psicosomáticos”, en Belloch, A. et al. (eds.): Manual de psicopatología. Madrid: Interamericana.

Tobin, D. et al. (1989): "The hierarchical factor structure of the Coping Strategies Inventory". Cognitive Therapy and Research, 13 (4): 343-361.

Vallejo-Sánchez, B. y Pérez-García, A. (2015): "Positividad y afrontamiento en pacientes con trastorno adaptativo". Anales de psicología, 31 (2): 462-471.

Wennberg, A. et al. (2015): "Alzheimer's patient familial caregivers: A review of burden and interventions". Health \& Social Work, 40 (4): 162-169. 
Anexo 1. Puntuaciones medias para las categorías de las variables

\begin{tabular}{|c|c|c|c|c|c|c|c|c|}
\hline & REP & AUC & EEM & PSD & APS & REC & EVP & RES \\
\hline \multicolumn{9}{|l|}{ Género } \\
\hline Hombre & 20,5 & 8,4 & 14,8 & 16,9 & 15,5 & 15,4 & 11,4 & 13,1 \\
\hline Mujer & 22,2 & 9,4 & 17,4 & 19,4 & 18,6 & 16,9 & 13,0 & 13,8 \\
\hline \multicolumn{9}{|l|}{ Edad } \\
\hline 55 años o menos & 23,1 & 9,9 & 17,7 & 19,3 & 18,3 & 18,1 & 13,2 & 14,1 \\
\hline De 56 a 65 años & 19,5 & 9,8 & 16,4 & 18,0 & 17,0 & 15,8 & 15,3 & 13,2 \\
\hline De 66 a 75 años & 23,1 & 8,8 & 16,5 & 18,7 & 18,0 & 17,0 & 11,6 & 13,0 \\
\hline 76 o más años & 21,9 & 8,5 & 17,3 & 20,0 & 19,4 & 15,6 & 10,5 & 15,0 \\
\hline \multicolumn{9}{|l|}{ Estado Civil } \\
\hline Soltero/a & 25,0 & 24,0 & 22,0 & 25,0 & 17,0 & 23,0 & 16,0 & 23,0 \\
\hline Pareja de hecho & 22,2 & 9,0 & 16,7 & 18,5 & 18,0 & 16,6 & 12,6 & 13,8 \\
\hline Viudo/a & 19,3 & 9,0 & 17,4 & 22,6 & 19,0 & 17,0 & 13,6 & 11,9 \\
\hline \multicolumn{9}{|l|}{ Situación laboral } \\
\hline Trabaja & 20,8 & 11,3 & 16,3 & 19,5 & 17,6 & 15,8 & 15,4 & 14,8 \\
\hline No trabaja & 22,1 & 9,0 & 17,0 & 19,0 & 18,2 & 16,8 & 12,5 & 13,6 \\
\hline \multicolumn{9}{|l|}{ Nivel de estudios } \\
\hline Sin estudios & 22,4 & 10,2 & 18,3 & 20,9 & 18,9 & 16,3 & 11,9 & 14,3 \\
\hline Primarios & 22,0 & 8,4 & 16,0 & 17,9 & 17,9 & 17,4 & 13,8 & 14,5 \\
\hline Secundarios - Terciarios & 20,0 & 8,9 & 15,2 & 17,7 & 16,3 & 15,5 & 12,3 & 9,3 \\
\hline \multicolumn{9}{|l|}{ Composición familiar } \\
\hline Sólo Cónyuge & 22,0 & 9,9 & 17,4 & 18,9 & 17,0 & 15,8 & 13,5 & 15,4 \\
\hline Sólo Hijos & 21,3 & 8,0 & 17,9 & 20,7 & 19,0 & 17,5 & 12,0 & 13,2 \\
\hline Cónyuge e hijos (con o sin otros familiares) & 22,2 & 9,1 & 16,3 & 20,2 & 18,4 & 16,2 & 12,4 & 12,3 \\
\hline Cónyuge y otros familiares (sin hijos) & 20,6 & 6,4 & 16,4 & 11,5 & 20,3 & 18,4 & 11,8 & 11,8 \\
\hline Hijos y otros familiares (sin cónyuge) & 25,0 & 17,5 & 16,0 & 19,3 & 18,7 & 23,0 & 14,3 & 17,0 \\
\hline \multicolumn{9}{|l|}{ Relación de parentesco } \\
\hline Esposo/a & 22,1 & 8,6 & 17,5 & 19,0 & 19,3 & 17,1 & 11,9 & 15,2 \\
\hline Hijo/a & 20,7 & 8,2 & 17,2 & 19,1 & 17,6 & 15,4 & 13,6 & 12,0 \\
\hline Nuera/yerno & 23,9 & 10,1 & 16,6 & 18,9 & 17,8 & 18,1 & 12,8 & 14,7 \\
\hline Otros & 23,5 & 17,7 & 13,8 & 19,3 & 15,0 & 17,5 & 13,3 & 13,3 \\
\hline \multicolumn{9}{|l|}{ Ha afectado la relación familiar } \\
\hline Sí & 23,1 & 10,8 & 18,7 & 21,4 & 19,9 & 17,0 & 12,8 & 13,2 \\
\hline No & 21,0 & 7,9 & 15,4 & 16,7 & 16,5 & 16,4 & 12,7 & 14,2 \\
\hline
\end{tabular}




\begin{tabular}{|c|c|c|c|c|c|c|c|c|}
\hline & REP & AUC & EEM & PSD & APS & REC & EVP & RES \\
\hline \multicolumn{9}{|l|}{ Frec. relaciones sociales } \\
\hline Todos los días & 23,2 & 9,1 & 18,7 & 21,6 & 19,5 & 17,2 & 12,7 & 14,4 \\
\hline Varias veces a la semana & 22,3 & 7,1 & 17,3 & 17,7 & 19,3 & 17,2 & 11,9 & 12,3 \\
\hline Una vez a la semana & 19,3 & 13,6 & 19,0 & 19,1 & 16,6 & 15,4 & 13,3 & 14,9 \\
\hline Varias veces al mes & 20,8 & 7,0 & 15,2 & 18,4 & 18,2 & 21,3 & 13,5 & 12,8 \\
\hline Una vez al mes o menos & 21,4 & 10,7 & 12,3 & 15,8 & 13,8 & 14,3 & 13,5 & 13,8 \\
\hline \multicolumn{9}{|l|}{ Cuidado anteriormente } \\
\hline Sí & 22,1 & 9,1 & 17,3 & 19,1 & 18,4 & 16,9 & 12,7 & 14,1 \\
\hline No & 20,5 & 11,0 & 12,5 & 18,3 & 14,8 & 14,8 & 13,3 & 8,5 \\
\hline \multicolumn{9}{|l|}{ Tiempo que lleva cuidando } \\
\hline Un año o menos & 22,5 & 7,1 & 16,3 & 18,6 & 18,8 & 15,8 & 11,0 & 13,1 \\
\hline Entre 1 y 3 años & 21,5 & 8,4 & 15,6 & 14,4 & 17,4 & 16,0 & 15,0 & 15,5 \\
\hline Más de 3 años & 21,8 & 10,6 & 17,5 & 20,7 & 18,0 & 17,4 & 13,1 & 13,5 \\
\hline \multicolumn{9}{|l|}{ Ha recibido información } \\
\hline Sí & 22,8 & 9,7 & 17,9 & 19,7 & 18,7 & 16,9 & 13,9 & 14,2 \\
\hline No & 20,6 & 8,8 & 15,6 & 18,2 & 17,3 & 16,5 & 11,3 & 13,1 \\
\hline \multicolumn{9}{|l|}{ Género del enfermo } \\
\hline Hombre & 21,6 & 8,4 & 17,0 & 19,0 & 19,3 & 16,9 & 12,9 & 15,3 \\
\hline Mujer & 22,3 & 10,2 & 16,8 & 19,0 & 16,7 & 16,6 & 12,7 & 11,8 \\
\hline \multicolumn{9}{|l|}{ Edad del enfermo } \\
\hline 75 años o menos & 23,0 & 7,9 & 15,8 & 20,3 & 18,5 & 17,1 & 11,9 & 14,7 \\
\hline De 76 a 80 años & 20,9 & 8,6 & 17,9 & 16,6 & 17,1 & 16,1 & 11,9 & 13,6 \\
\hline De 81 a 85 años & 22,3 & 12,0 & 17,1 & 20,1 & 18,9 & 17,5 & 14,7 & 14,1 \\
\hline 86 años o más & 20,6 & 8,6 & 17,1 & 18,7 & 17,8 & 15,6 & 12,6 & 11,3 \\
\hline \multicolumn{9}{|l|}{ Nivel de deterioro } \\
\hline Leve & 20,7 & 6,7 & 16,7 & 18,0 & 19,8 & 17,1 & 12,0 & 15,4 \\
\hline Moderado & 21,5 & 8,8 & 15,4 & 18,0 & 17,1 & 16,3 & 12,4 & 12,8 \\
\hline Grave & 23,0 & 10,8 & 19,2 & 20,6 & 18,9 & 17,2 & 13,5 & 14,2 \\
\hline
\end{tabular}

Fuente: Elaboración propia. 